\title{
Doble pantalla todo el año: Interacción en torno a las cuentas de Twitter de periodistas televisivos en México
}

Antonio Corona | antoniorir@gmail.com Universidad Autónoma de Coahuila

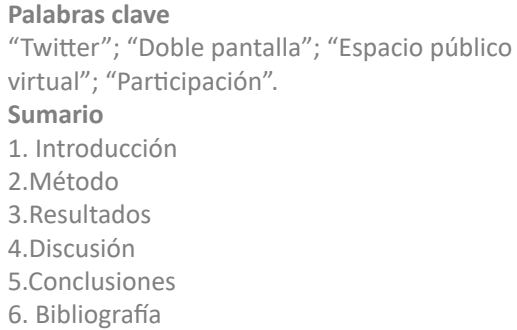

\section{Resumen}

El presente trabajo recuperó un año natural de actividad en torno a las cuentas personales de 20 periodistas con alta presencia televisiva en México, con la intención de esclarecer si dicha actividad se relaciona con la aparición de los periodistas en medios tradicionales, lo cual indicaría prácticas de doble pantalla como las que se han estudiado durante momentos de alta mediatización política. Se categorizó la actividad de acuerdo a horas del día, y se graficó comparativamente la distribución porcentual de la actividad de envío y recepción, teniendo en cuenta los horarios de aparición mediática de los periodistas. Se encontró que la interacción del público con los periodistas fue consecuencia de la aparición de los mismos en medios tradicionales, más que de su actividad en Twitter. Los resultados nos indican que la actividad en Twitter alrededor de los periodistas no existe en un vacío, sino que es parte de un ambiente mediático híbrido en el cual Twitter es utilizado como una herramienta de contestación de las enunciaciones en los noticieros televisivos. Se concluye que las prácticas de doble pantalla son un fenómeno de la cotidianeidad, y no sólo de momentos de alta mediatización como los debates electorales, y que esto debe reflejarse en la investigación sobre el fenómeno.

\section{Cómo citar este texto:}

Antonio Corona (2019): Doble pantalla todo el año: Interacción en torno a las cuentas de Twitter de periodistas televisivos en México, en Miguel Hernández Communication Journal, n¹0 (1), pp. 199 a 221. Universidad Miguel Hernández, UMH (Elche-Alicante). DOI: http://dx.doi.org/10.21134/mhcj.v10i0.291 


\title{
Dual Screen all year long: Interactions around TV journalists' Twitter accounts in Mexico
}

\author{
Antonio Corona | antoniorir@gmail.com \\ Universidad Autónoma de Coahuila
}

\begin{abstract}
Keywords "Participation".

Summary

1. Introduction

2. Method

3. Results

4. Discussion

5. Conclusions

6. Bibliography
\end{abstract}

\section{Abstract}

"Twitter"; "Dual screening"; "Virtual public space”,

The present work recovered a natural year of activity

around the personal accounts of 20 highly-televised journalists in Mexico, to determine whether this activity was related to their appearance on traditional media, which would indicate practices of dual screening such as the ones that have been previously studied during moments of high political mediatization. The activity was categorized, and the hourly percentages of publication and interaction were visually compared to each other and to the journalists' appearances on the media. It was found that audience interaction with the journalists was the result of their appearance on the media, rather than of their activity on Twitter. These results indicate that Twitter activity around journalists doesn't exist in a vacuum, but rather in a hybrid media environment in which Twitter is used as a tool of enunciation aimed at the TV. It's concluded that dual screening practices are a phenomenon of the everyday life, and not just of highly-mediatized moments such as political debates, and that this should be reflected in our research about this phenomenon.

\section{How to cite this text}

Antonio Corona (2019): Dual Screen all year long: Interactions around TV journalists' Twitter accounts in Mexico, in Miguel Hernández Communication Journal, n¹0 (1), pp. 199 a 221. Universidad Miguel Hernández, UMH (Elche-Alicante). DOI: http://dx.doi.org/10.21134/mhcj.v10i0.291 


\section{Introducción}

La aparición de Twitter fue recibida por la comunidad académica con optimismo respecto a su potencial para alentar y posibilitar la participación ciudadana en el diálogo de la esfera pública ${ }^{1}$. Sin embargo, tanto las circunstancias seculares como los estudios subsecuentes nos dieron hace ya tiempo motivos para moderar nuestras esperanzadoras apreciaciones iniciales; en vez del adviento de la "esfera pública digital” y su participación simétrica y trascendente, nos encontramos con panoramas de alta polarización y encapsulación en cámaras de eco, donde el diálogo entre posturas divergentes es mínimo o nulo (Barberá et al, 2015; Colleoni, Rozza y Arvidsson, 2014; Conover et al, 2011; Gruzd y Roy, 2014; Morales et al, 2015; Yardi y Boyd, 2010).

Ante dichos panoramas, el optimismo indiscriminado respecto a Twitter como plataforma de ejercicio de la ciudadanía ha cedido el campo a estudios más mesurados sobre las circunstancias específicas en las cuales dichas plataformas se convierten en avenidas de agencia para los ciudadanos. Uno de los puntos a los que más se ha dado atención en este sentido es a la relación entre la actividad de corte político en Twitter y la recepción y circulación de noticias. Específicamente, se ha estudiado la manera en que los usuarios utilizan Twitter como plataforma para discutir eventos noticiosos al tiempo que los observan en televisión. A este fenómeno de consumo mediático se le ha llamado "doble pantalla" o "segunda pantalla", y sus proponentes argumentan que esta manera de utilización de la red social es un predictor confiable de involucramiento y participación política tanto a corto como a largo plazo.

Nos referimos como doble pantalla o segunda pantalla al "conjunto de prácticas que involucran la integración -y movimiento entre- los medios de transmisión en vivo y las redes sociales.” (Vaccari, Chadwick y O'Loughlin, 2015 p. 1041). Es decir, la articulación entre la recepción del contenido de los medios formales y la interacción en redes sociales con los presentadores de dicho contenido (sea en forma de pregunta, denuncia o elogio, por ejemplo) para dar lugar a un consumo mediático más activo, en el cual la contestación o pronunciamiento en redes sociales constituye una avenida de agencia del receptor que puede, incluso, reducir la asimetría de las relaciones de producción mediática (Chadwick, 2017; Chadwick, O’Loughlin y Vaccari, 2017).

No hay aún consenso respecto a la terminología para referirnos a este fenómeno: se le llama segunda pantalla (Giglietto y Selva, 2014; De Zúñiga y Liu, 2017), doble pantalla (Shah et al, 2016; Vaccari, Chadwick y O’Loughlin, 2015) o términos más específicos pero que irónicamente pueden resultar más confusos, como "sistema mediático híbrido" (Chadwick, 2017; Giglietto et al, 2016). En el presente trabajo se utilizarán estos términos de manera intercambiable, para evitar favorecer a un término específico.

Si bien el concepto de la múltiple pantalla está ampliamente desarrollado en el campo del marketing, no es el empleo de pantallas múltiples para aumentar el consumer engagement lo que nos llama la atención como científicos sociales, sino las consecuencias y significado de estas prácticas emergentes para nuestro entendimiento de la manera en que Twitter es o puede llegar a ser una avenida de agencia y enunciación para los ciudadanos. Este fenómeno ha sido investigado a profundidad en los últimos años, pero las prácticas de segunda o doble pantalla se han estudiado principalmente alrededor de eventos de alta participación política, como es el caso de los debates electorales televisados (De Zúñiga y Liu, 2017; Vaccari, Chadwick y O'Loughlin, 2015).

${ }^{1}$ Las esperanzadoras conclusiones de Bruns y Highfield (2012), Effing, van Hillegersberg y Huibers (2011) y Loader y Mercea (2012) son ejemplos de dicho optimismo. 
Es innegable la utilidad de analizar fenómenos de participación durante momentos en los que se sabe que hay una participación inusitadamente alta: el aumento de actividad visibiliza las lógicas, patrones y comportamientos cuya presencia tal vez fuera demasiado sutil para identificarse en otro momento; por otro lado, si lo que se busca (como en el caso de la "doble pantalla") es comprender la cantidad y calidad de la participación, estudiar solamente los momentos de más alta participación genera un gran punto ciego metodológico.

Ante esta situación, el objetivo del presente estudio fue analizar la posible presencia de prácticas de "doble pantalla" en los ciclos noticiosos mediáticos de la cotidianeidad, mediante el análisis de un año natural de actividad alrededor de las cuentas de 20 de los presentadores de noticias más influyentes de México en medios tradicionales. Este tipo de análisis puede darnos más información respecto a las dimensiones de la relación entre los medios noticiosos tradicionales y la participación en Twitter durante la vida diaria, y no solamente durante los eventos y épocas de politización atípicamente alta.

Con esta finalidad, se analizó la distribución horaria de las publicaciones (tweets) de las cuentas de los periodistas. Se hizo lo mismo con las publicaciones del público en general que mencionaron o fueron dirigidas a estos periodistas, y ambas distribuciones se compararon con los horarios de transmisión de los noticieros televisivos y radiofónicos que cada uno de los periodistas. Se llevó a cabo esta comparación con la finalidad de esclarecer si la interacción del público con las cuentas de los periodistas obedece a la actividad de la cuenta en sí misma, o a la actividad del periodista en medios tradicionales.

Respecto a los resultados, se previeron 3 escenarios posibles:

1. Que exista una relación entre las distribuciones de publicación de las cuentas de los periodistas y la interacción del público con éstas, independientemente de los horarios de transmisión. Esto cual implicaría que la interacción en Twitter alrededor de estas cuentas se debe a la actividad de la misma cuenta y no a la actividad del periodista en medios tradicionales, poniendo en duda la existencia de prácticas de doble pantalla en la cotidianeidad.

2. Que exista una relación constante entre publicación, interacción y horarios de transmisión, lo cual impediría formular conclusiones, debido a la imposibilidad de aislar las variables.

3. Que exista una relación entre la interacción en torno a la cuenta y la transmisión de los noticieros, independientemente de la actividad de la cuenta misma, lo cual indicaría la existencia de hibridación entre el consumo mediático de noticias tradicionales y la expresión de opiniones en Twitter (es decir, un fenómeno de doble pantalla) en el ciclo de consumo de noticias de la vida cotidiana, y no solamente en momentos de alta mediatización o politización, en los cuales se ha centrado casi toda la investigación respecto al fenómeno.

4. Que no exista relación alguna de la interacción del público ni con la actividad de la cuenta ni con la transmisión de noticieros, lo cual apuntaría hacia la inexistencia de hibridación mediática (es decir, de prácticas de múltiple pantalla) en la vida cotidiana.

\section{Método}

El presente reporte se desprende del proyecto de investigación "Identidad, ciudadanía y pertenencia en la recepción y circulación de contenido informativo en Twitter”, cuya finalidad es conocer y comprender el papel que han jugado los distintos procesos identitarios en la creciente desconfianza hacia el periodismo formal y las fuentes establecidas de producción de contenido 
informativo, y en la proliferación y masificación del contenido noticioso informal, surgimiento de cámaras de eco, apelaciones masivas a la emoción y otros fenómenos similares que hoy en día son agrupados bajo el término paraguas de "post-verdad" (Lockie, 2016; Suiter, 2016). Dicho proyecto consiste de cuatro focos de análisis: Involucramiento, Doble Pantalla, Otrificación y Post-Verdad. En este reporte se presentan resultados de la segunda etapa, correspondiente al análisis cuantitativo de la manera en que se relaciona la producción de enunciaciones en Twitter con la recepción de contenidos noticiosos formales.

Vaccari, Chadwick y O’Loughlin (2015) mencionan que el conjunto de prácticas al que nos referimos como segunda o doble pantalla es inusual y emergente (aunque el mismo Chadwick en su libro subsecuente afirma que la hibridez es y ha sido siempre una característica de todo panorama mediático), por los cual "explicar su repercusión en el comportamiento político requiere innovación metodológica” (p. 1041). En lo que se refiere a la formulación de métricas y metodologías para la investigación cuantitativa en Twitter, Bruns y Stieglitz (2014) mencionan que, si bien la extracción de datos mediante API posibilita (y hasta cierto punto estandariza) la extracción de métricas claves, el uso y entendimiento efectivo de dichas cifras siempre dependerá de un diseño metodológico apropiado para cada investigación.

Dichos autores presentan cuatro categorías de las métricas que pueden ser obtenidas de la extracción sistemática de datos en Twitter: métricas básicas, temporales, de usuario y de agrupación. En cuanto a la agenda de investigación, Williams, Terras y Warwick (2013) clasifican el estudio académico de Twitter de acuerdo al objeto de investigación, presentando cuatro categorías posibles: estudio del mensaje, del usuario, de la tecnología y del concepto. Desde el punto de vista de la comunicación, nuestra atención recae principalmente en el estudio del mensaje y del usuario. De esto se desprende que, en la matriz resultante de las clasificaciones de Bruns y Stieglitz (2014) y Williams, Terras y Warwick (2013), la presente investigación en su etapa actual corresponde a un estudio del mensaje utilizando métricas básicas, temporales y de usuario. El método empleado tanto para la recuperación como para el análisis de los datos obtenidos es un reflejo de esta delimitación metodológica, y está informado por la misma.

Como primer paso para conformar la base de datos que se analizó en este estudio, se realizó un censo de los noticieros televisivos de transmisión nacional al 1 de Enero del 2017. No se tomaron en cuenta para la elaboración de esta parrilla los noticieros de corte local o metropolitano, aun cuando su distribución fuera semi-nacional o nacional. Con la información recabada se elaboró una parrilla de noticieros televisivos nacionales en México (tabla 1).

Subsecuentemente, se recuperaron las cuentas personales de aquellos presentadores televisivos de noticias a nivel nacional cuyos noticieros no cuentan con una cuenta propia, por lo cual las interacciones en Twitter relacionadas con el noticiero serían dirigidas, presumiblemente, a la cuenta personal del periodista titular.

Se decidió tomar este criterio para disminuir la posibilidad de incertidumbre: en el contexto de las prácticas de múltiple pantalla, asumimos que el usuario utiliza Twitter para responder a una enunciación vista en televisión o escuchada en radio. En este sentido, analizar cuentas de periodistas cuyos noticieros no tienen cuenta propia disminuye la posibilidad de que la respuesta del usuario se dirija a la cuenta del noticiero y no a la del periodista. Resultaron de este ejercicio 20 cuentas personales de periodistas conocidos a nivel nacional, como se muestra en la tabla 2. 
Tabla 1. Parrilla de programación, noticieros nacionales. Fuente: Elaboración propia.

\begin{tabular}{|c|c|c|c|c|}
\hline Programa & Horario & Canal & Cuenta & Presentador \\
\hline $\begin{array}{l}\text { Las Noticias } \\
\text { Despierta con Loret } \\
\text { Al Aire con Paola Rojas } \\
\text { El Noticiero } \\
\text { En Punto }\end{array}$ & $\begin{array}{l}6: 00 \\
6: 30 \\
8: 00 \\
14: 30 \\
22: 00\end{array}$ & $\begin{array}{l}\text { Las Estrellas } \\
\text { Las Estrellas } \\
\text { Las Estrellas } \\
\text { Las Estrellas } \\
\text { Las Estrellas }\end{array}$ & $\begin{array}{l}\text { “@daniellemx_” } \\
\text { "@CarlosLoret” } \\
\text { "@Paola_Rojas_H” } \\
\text { “@karlaiberia”" } \\
\text { "@DeniseMaerker" }\end{array}$ & $\begin{array}{l}\text { Danielle Dithurbide } \\
\text { Carlos Loret de Mola } \\
\text { Paola Rojas } \\
\text { Karla Iberia } \\
\text { Denise Maerker }\end{array}$ \\
\hline $\begin{array}{l}\text { Las Noticias } \\
\text { Matutino Express } \\
\text { Las Noticias } \\
\text { Agenda Pública } \\
\text { En una Hora } \\
\text { Hora } 21 \\
\text { Es Hora de Opinar }\end{array}$ & $\begin{array}{l}6: 00 \\
8: 00 \\
14: 30 \\
19: 30 \\
20: 00 \\
21: 00 \\
22: 00\end{array}$ & $\begin{array}{l}\text { Foro TV } \\
\text { Foro TV } \\
\text { Foro TV } \\
\text { Foro TV } \\
\text { Foro TV } \\
\text { Foro TV } \\
\text { Foro TV }\end{array}$ & $\begin{array}{l}\text { “@ErikCamachoTV” } \\
\text { “@Ftv_MatutinoEx" } \\
\text { “@Foro_TV” } \\
\text { "@mariocampos” } \\
\text { “@guillermochoa” } \\
\text { "@patojulian” } \\
\text { “@leozuckermann” }\end{array}$ & $\begin{array}{l}\text { Mario Campos } \\
\text { Guillermo Ochoa Jr. } \\
\text { Julio Patán } \\
\text { Leo Zuckermann }\end{array}$ \\
\hline $\begin{array}{l}\text { Hechos AM } \\
\text { Hechos Meridiano } \\
\text { Hechos Noche }\end{array}$ & $\begin{array}{l}6: 00 \\
14: 00 \\
20: 00\end{array}$ & $\begin{array}{l}\text { Azteca Uno } \\
\text { Azteca Uno } \\
\text { Azteca Uno }\end{array}$ & $\begin{array}{l}\text { "@HechosAM" } \\
\text { "@Hmeridiano" } \\
\text { "@NoticieroHechos" }\end{array}$ & TV Azteca \\
\hline $\begin{array}{l}\text { Noticias de Ida y Vuelta } \\
\text { Informativo } 40 \text { con Lilly } \\
\text { Tellez } \\
\text { Informativo } 40 \text { con Hannia } \\
\text { Novell }\end{array}$ & $\begin{array}{l}14: 30 \\
15: 00 \\
20: 00\end{array}$ & $\begin{array}{l}\text { Azteca } 40 \\
\text { Azteca } 40 \\
\text { Azteca } 40\end{array}$ & $\begin{array}{l}\text { “@MarioBeteta” } \\
\text { “@LillyTellez” } \\
\text { “@HanniaNovell” }\end{array}$ & $\begin{array}{l}\text { Oscar Mario Beteta } \\
\text { Lilly Téllez } \\
\text { Hannia Novell }\end{array}$ \\
\hline $\begin{array}{l}\text { Imagen Noticias con Fran- } \\
\text { cisco Zea } \\
\text { Imagen Noticias con Yuriria } \\
\text { Sierra } \\
\text { Imagen Noticias con Ciro } \\
\text { Gómez Leyva }\end{array}$ & $\begin{array}{l}6: 00 \\
14: 00 \\
22: 00\end{array}$ & $\begin{array}{l}\text { Imagen Televi- } \\
\text { sión } \\
\text { Imagen Televi- } \\
\text { sión } \\
\text { Imagen Televi- } \\
\text { sión } \\
\end{array}$ & $\begin{array}{l}\text { “@franciscozea” } \\
\text { “@YuririaSierra” } \\
\text { “@CiroGomezL” }\end{array}$ & $\begin{array}{l}\text { Francisco Zea } \\
\text { Yuriria Sierra } \\
\text { Ciro Gómez Leyva }\end{array}$ \\
\hline $\begin{array}{l}\text { México al día AM } \\
\text { México al día MD } \\
\text { Noticias } 22 \\
\text { México al día PM }\end{array}$ & $\begin{array}{l}7: 30 \\
14: 30 \\
19: 00 \\
20: 30\end{array}$ & $\begin{array}{l}\text { Canal } 22 \\
\text { Canal } 22 \\
\text { Canal } 22 \\
\text { Canal } 22\end{array}$ & $\begin{array}{l}\text { “@mexaldia” } \\
\text { "@mexaldia" } \\
\text { "@laurabarrera99" } \\
\text { “@mexaldia" }\end{array}$ & Laura Barrera \\
\hline $\begin{array}{l}\text { Once Noticias con Javier } \\
\text { Solórzano } \\
\text { Once Noticias con Gpe. } \\
\text { Contreras } \\
\text { Once Noticias con Adriana } \\
\text { P. Cañedo }\end{array}$ & $\begin{array}{l}6: 00 \\
12: 30 \\
21: 00\end{array}$ & $\begin{array}{l}\text { Canal Once } \\
\text { Canal Once } \\
\text { Canal Once }\end{array}$ & $\begin{array}{l}\text { “@JavierSolorzano” } \\
\text { “@lumiery” } \\
\text { “@aperezcanedo” }\end{array}$ & $\begin{array}{l}\text { Javier Solórzano } \\
\text { Guadalupe Contreras } \\
\text { Adriana P. Cañedo }\end{array}$ \\
\hline
\end{tabular}

Se realizó una recopilación sistemática durante un año natural (2017), capturando todas las publicaciones realizadas por las cuentas de la tabla 2, así como todas las publicaciones dirigidas a -o mencionando a- alguna de estas cuentas. Dicha recopilación se realizó mediante requisiciones de pesquisa (queries) al API de Twitter, utilizando el lenguaje de programación Python (Python Software Foundation, 2010) en su distribución Anaconda (Continuum Analytics, 2012) y, más específicamente, el protocolo Twython, diseñado por Ryan McGrath (2013) para la interacción con el API de dicha red social. Cada uno de los productos de software arriba mencionados son distribuidos bajo el esquema de código abierto (Open Source).

Utilizando estas herramientas, se recuperaron las publicaciones realizadas por cuentas de la muestra y las publicaciones dirigidas a cuentas de la muestra con una frecuencia diaria, quincenal o semanal, dependiendo de la densidad de actividad histórica de cada una de las cuentas. Es 
posible que, debido a error humano o a problemas de comunicación, en algunos casos no se haya conseguido capturar el total absoluto de la actividad; sin embargo, dado que el volumen de las publicaciones recuperadas es tan amplio, y el posible volumen de las publicaciones no recuperadas tan pequeño, es nuestra convicción que no hay ninguna diferencia apreciable ni humana ni estadísticamente entre la muestra recuperada y el universo de la actividad en torno a estas cuentas.

Tabla 2. Cuentas observadas. Fuente: Elaboración propia.

\begin{tabular}{|l|l|l|l|}
\hline Periodista & Cuenta & Periodista & Cuenta \\
\hline Adriana Pérez Cañedo & @aperezcanedo & Karla Iberia & @Karlaiberia \\
\hline Carlos Loret de Mola & @CarlosLoret & Laura Barrera & @laurabarrera99 \\
\hline Ciro Gómez Leyva & $@$ CiroGomezL & $\begin{array}{l}\text { Leo Zucker- } \\
\text { mann }\end{array}$ & @leozuckermann \\
\hline Danielle Dithurbide & @, daniellemx_ & Lilly Téllez & @LillyTellez \\
\hline Denise Maerker & @DeniseMaerker & $\begin{array}{l}\text { Guadalupe } \\
\text { Contreras }\end{array}$ & @lumiery \\
\hline Erik Camacho & @ErikCamachoTV & Mario Beteta & @MarioBeteta \\
\hline Francisco Zea & @Franciscozea & Mario Campos & @mariocampos \\
\hline Guillermo Ochoa & @guillermochoa & Paola Rojas & @Paola_Rojas_H \\
\hline Hannia Novell & @HanniaNovell & Julio Patán & @patojulian \\
\hline Javier Solórzano & $@$ JavierSolorzano & Yuriria Sierra & $@$ YuririaSierra \\
\hline
\end{tabular}

En el contexto de este trabajo, se denomina "publicaciones fuente" o simplemente "publicaciones" a los Tweets realizados por las cuentas observadas - las pertenecientes a los periodistas de la tabla 2-, y se denomina "interacciones" a las publicaciones realizadas por cuentas del público en general -es decir, cualquier cuenta que no esté prevista en la tabla 2- cuyo objeto fue una o más de las cuentas observadas. Esta interacción puede tomar la forma de:

1. Respuesta directa a una publicación de una cuenta observada.

2. Publicación dirigida a una cuenta observada, que no responde a una publicación específica.

3. Respuesta directa a publicación de una cuenta no observada, cuyo texto menciona a una cuenta observada.

4. Publicación dirigida a una cuenta no observada, cuyo texto menciona a una cuenta observada.

5. Publicación no dirigida a una cuenta específica, cuyo texto menciona a una cuenta observada.

Esta categorización no contempla como interacción la figura de retweet, debido a que la mecánica del retweet no implica enunciación, sino simplemente un acto mecánico similar al like de Facebook u otras mecánicas equivalentes en distintas redes sociales. En Bruns y Stieglitz (2014) se puede leer más a fondo respecto a la diferencia cualitativa entre el acto de retweet (lo que ellos llaman aproximación diseminativa) y una interacción formal en la forma de mención o respuesta (lo que llaman aproximación enunciativa y/o discursiva). En ese sentido, si bien se podría argumentar que el retweet es una forma de interacción en el aspecto mecánico, no lo es desde un punto de vista enunciativo. 
Esta distinción es importante cuando el fenómeno estudiado tiene que ver con la participación e involucramiento, como es el caso del fenómeno de múltiple pantalla que estudiamos aquí, por lo cual se tomó la decisión metodológica de no tomar en cuenta los retweets para efectos de esta investigación.

\section{Resultados}

En la tabla 3 podemos apreciar los números brutos de la actividad del año 2017 por horas en torno a las cuentas observadas. Con la finalidad de esclarecer la relación entre los indicadores, se obtuvo la proporción porcentual (multiplicando el total anual de publicaciones de cada hora por $100 \mathrm{y}$ dividiendo entre el total de publicaciones en ese rubro). Esta operación nos permite referirnos a la actividad en cuanto a porcentajes horarios de un total de tráfico anual, y con ello observar de una manera mucho más clara tanto la relación que las publicaciones de las cuentas observadas tienen con las interacciones que generan, como la relación que ambas tienen con la programación mediática. Las figuras 1 a la 20 corresponden a las graficaciones de los porcentajes horarios de actividad de cada una de las cuentas observadas, tanto en publicaciones como en interacciones.

Tabla 3. Desglose de volumen: publicaciones e interacciones.

Fuente: Elaboración propia.

\begin{tabular}{|l|l|l|l|l|l|}
\hline Cuenta & Publicaciones & $\begin{array}{l}\text { Interac- } \\
\text { ciones }\end{array}$ & Cuenta & Publicaciones & $\begin{array}{l}\text { Interaccio- } \\
\text { nes }\end{array}$ \\
\hline aperezcanedo & 2494 & 4285 & karlaiberia & 3046 & 13253 \\
CarlosLoret & 6759 & 229608 & laurabarrera99 & 495 & 1927 \\
CiroGomezL & 11653 & 182602 & leozuckermann & 264 & 22206 \\
daniellemx_ & 1470 & 32605 & LillyTellez & 1031 & 11331 \\
DeniseMaerker & 16611 & 192804 & lumiery & 295 & 405 \\
ErikCamachoTV & 713 & 2093 & MarioBeteta & 20541 & 29603 \\
franciscozea & 2985 & 46808 & mariocampos & 2809 & 29030 \\
guillermochoa & 2019 & 8025 & Paola_Rojas_H & 351 & 6916 \\
HanniaNovell & 942 & 13380 & patojulian & 774 & 5055 \\
JavierSolorzano & 1026 & 10239 & YuririaSierra & 2746 & 50681 \\
\hline
\end{tabular}

$\mathrm{Al}$ realizar las graficaciones mencionadas, surgieron 4 grupos de casos: en el primero, los picos de frecuencia de interacciones coinciden con los de publicaciones; es decir, la hora del día con más menciones y respuestas a la cuenta observada es también la hora del día en la que dicha cuenta tiene más publicaciones (figuras 1 a la 6). Tal es el caso de Erik Camacho y Julio Patán del canal noticioso Foro TV, así como de Hannia Novell y Lilly Téllez de Informativo 40, Guadalupe Contreras, , Karla Iberia del Canal de Las Estrellas y Guadalupe Contreras, de Canal 22. Si bien hay diferencias amplias de volumen tanto de publicaciones como de interacciones entre las cuentas de estos periodistas (la de Guadalupe Contreras, por ejemplo, sólo fue objeto de 405 interacciones en el año), este comportamiento está presente de modo similar en todas las cuentas mencionadas. Al comparar estas frecuencias de interacción y publicación con las horas de transmisión de los respectivos noticieros que presentan los periodistas, nos encontramos con que no sólo coinciden los picos de interacción y publicación, sino que ambos coinciden con la hora de transmisión de los noticeros. 
Figura 1. Actividad por hora, 2017: Erik Camacho. Fuente: Elaboración propia.

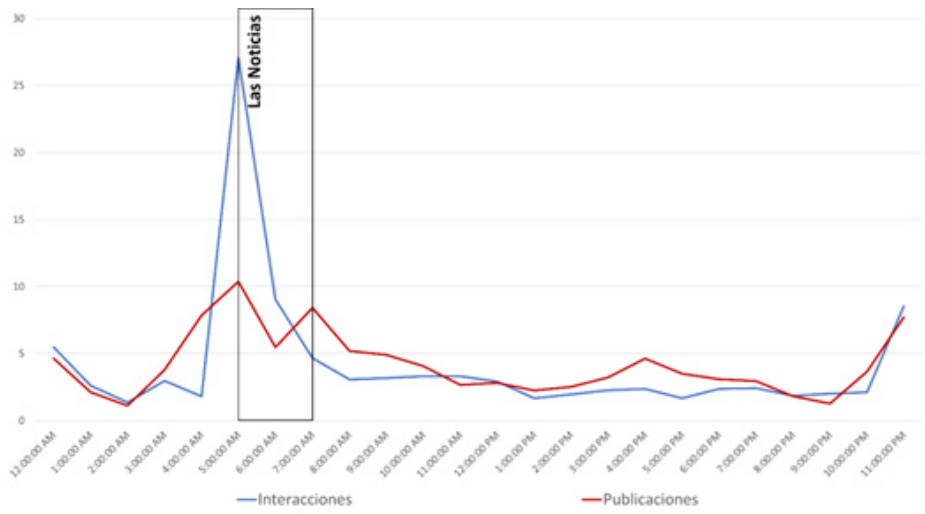

Figura 2. Actividad por hora, 2017: Guadalupe Contreras. Fuente: Elaboración propia.

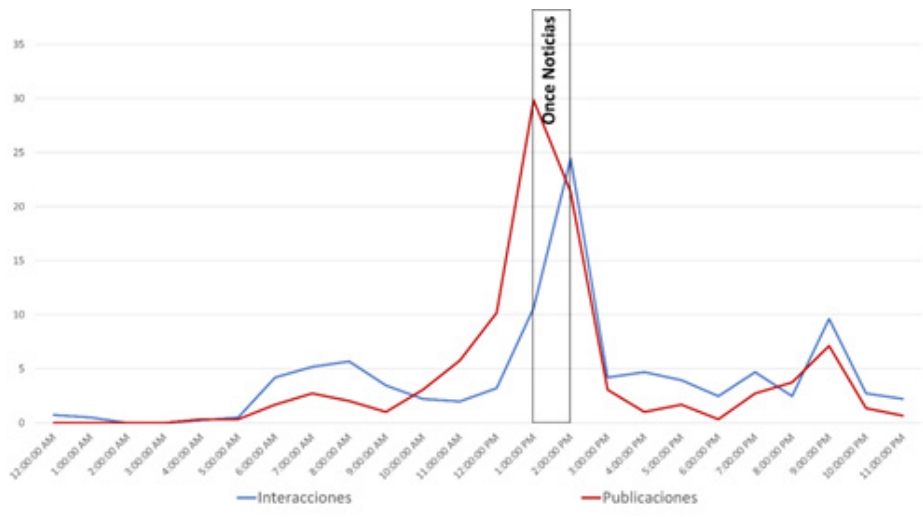

Figura 3. Actividad por hora, 2017: Karla Iberia. Fuente: Elaboración propia.

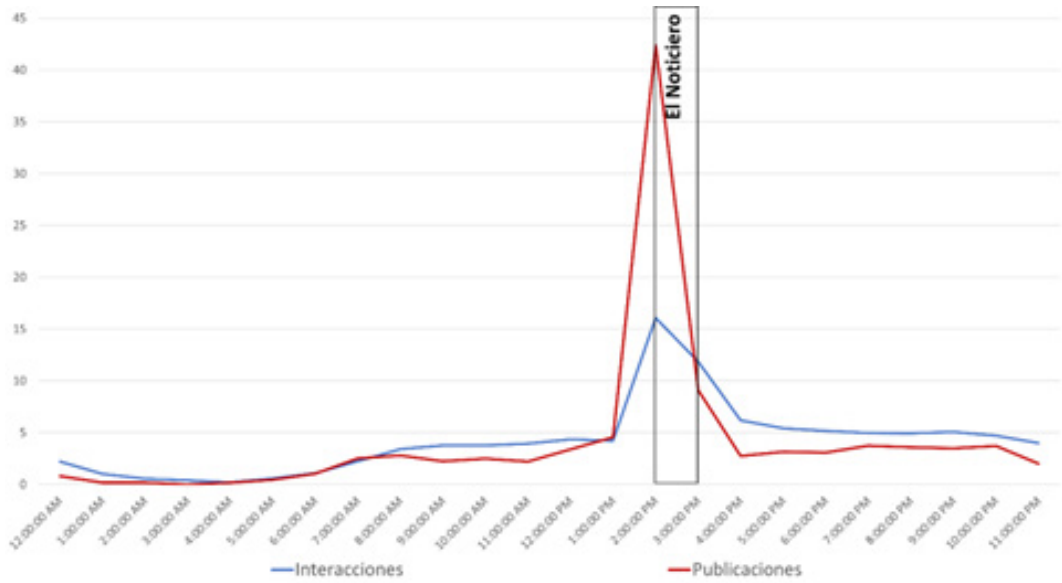


Figura 4. Actividad por hora, 2017: Hannia Novell. Fuente: Elaboración propia.

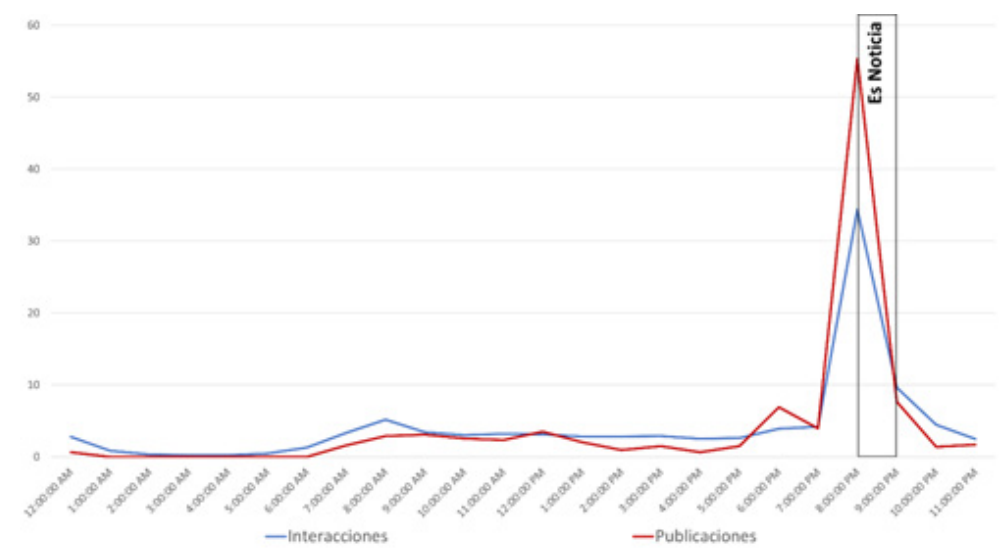

Los siguientes tres periodistas (figuras 7 a la 9) tienen cada uno dos programas diferentes de transmisión nacional: Mario Campos aparece 3 horas por la mañana en Antena Radio, y conduce el programa de análisis "Agenda Pública” por la noche. Yuriria Sierra y Francisco Zea, por su parte, conducen tanto noticieros televisivos como radiofónicos en Grupo Imagen. En el caso de estos dos últimos, el horario de transmisión de sus respectivos programas coincide también con los picos más altos de actividad en publicaciones y en interacciones. El caso de Mario Campos (figura 9) es diferente: sus horas de mayor actividad de publicación son similares en volumen $(10.32 \%$ a las 7 a.m. y $11.09 \%$ a las 8 p.m.) y además coinciden con los horarios de sus programas; sin embargo, durante su programa radiofónico matutino no hay un pico de interaccciones como en el programa nocturno (6.12\% a las 7 a.m. contra $12.81 \%$ a las 8:00 p.m.) como se puede apreciar en la figura 9.

Algo similar sucede con las cuentas de Ciro Gómez Leyva (figura 10), Carlos Loret de Mola (figura 11), Denise Maerker (figura 12) y Javier Solórzano (figura 13): cada uno de estos periodistas es figura de dos programas diferentes, y en cada uno de los casos, la cuenta del periodista publica más durante el programa, pero sólo uno de sus dos programas va acompañado de un pico en las interacciones. La cuenta de Ciro Gómez Leyva publica mucho más durante su noticiero televisivo $(36.58 \%)$ que durante el noticiero radiofónico matutino (12.13\%), pero en ambos casos es objeto de un número similar de interacciones (10.84\% y $11.22 \%$ ), e incluso recibe un mayor volumen de interacciones durante su aparición en radio. La cuenta de Carlos Loret, el periodista más popular de los 20 observados por número de inteacciones, tiene dos momentos de alta actividad, que significan cada uno el 20\% del total de publicación. Como sucede con los periodistas anteriores, los picos de publicación corresponden directamente a sus programas de televisión (Despierta con Loret) y de radio (Contraportada). Se puede observar, sin embargo, que las interacciones con la cuenta por parte del público no comparten esa simetría, sino que son más frecuentes durante la transmisión del programa televisivo (12.06\%, contra 6.61\% durante el programa radiofónico).

El "noticiero cool" de Javier Solórzano, que se basa fuertemente en la interacción a través de redes sociales, genera una gran cantidad de mensajes en comparación con la aparición del periodista en el noticiero matutino de Canal Once (figura 13). La interacción por parte del público, sin embargo, ignora esta distribución, y presenta una distribución acorde a los horarios de transmisión, con mayor porcentaje de interacción durante su noticiero tradicional. 
El siguiente grupo de casos está compuesto por Guillermo Ochoa Jr. (figura 14), Leo Zuckermann (figura 15)y Paola Rojas (figura 16), todos ellos periodistas de la cadena Televisa. En estos casos, los periodistas en cuestión tienen una amplia actividad en Twitter fuera de los horarios de sus noticieros, lo cual se refleja en los picos de las gráficas. Sin emargo, como se puede observar en las mismas gráficas, la distribución de las interacciones no sigue a la de las publicaciones, sino a los horarios de transmisión. Es decir: aún cuando estos periodistas publican más tweets fuera de sus horarios de transmisión, las menciones y mensajes dirigidos a sus cuentas siguen teniendo sus auges durante los noticieros.

Figura 5. Actividad por hora, 2017: Julio Patán. Fuente: Elaboración propia.

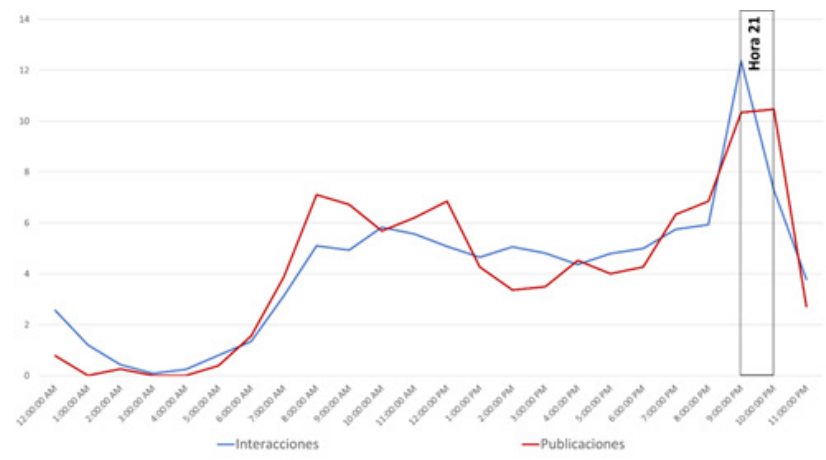

Figura 6. Actividad por hora, 2017: Lilly Téllez. Fuente: Elaboración propia.

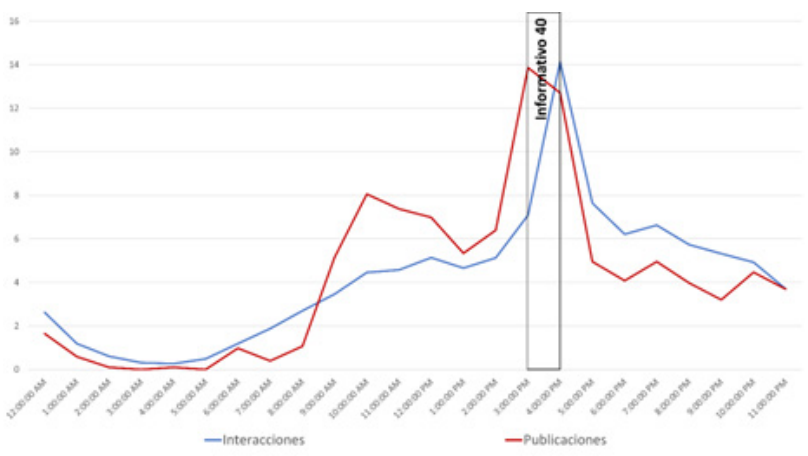

Figura 7. Actividad por hora, 2017: Yuriria Sierra. Fuente: Elaboración propia.

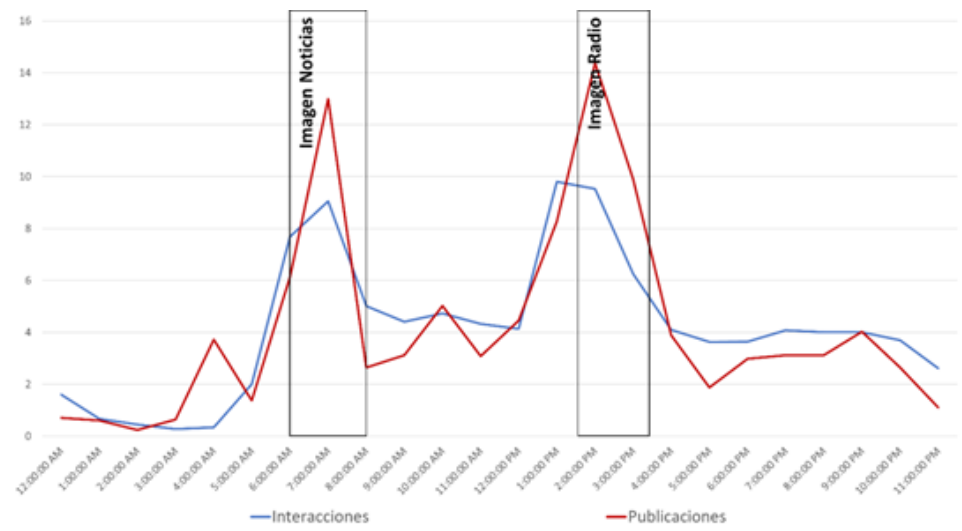


Figura 8. Actividad por hora, 2017: Francisco Zea. Fuente: Elaboración propia.

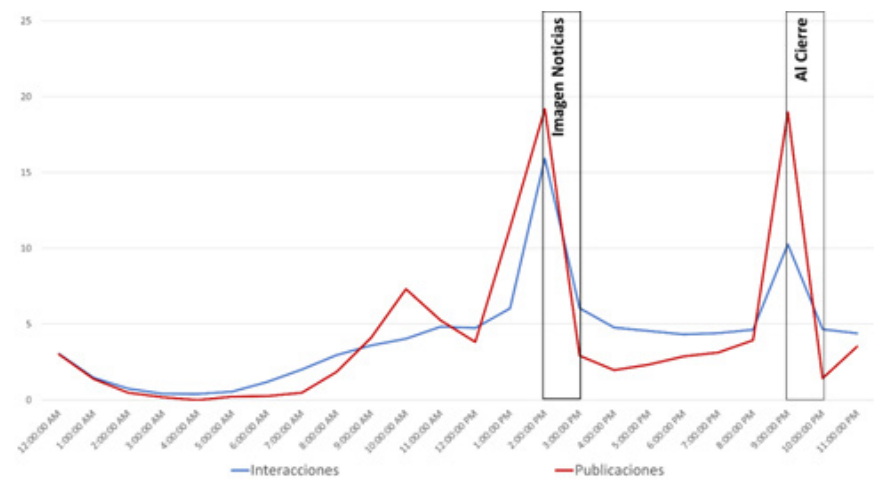

La figura 16 corresponde a la cuenta de Paola Rojas, quien aparece de Lunes a Viernes a las 8 a.m en Las Estrellas como presentadora del noticiero "Al Aire con Paola Rojas", y en Radio Fórmula de 5 a 6 de la tarde. Como se observa en la figura 16, la mayor cantidad de interacciones con la cuenta de esta periodista se da de las 8 a las 9 am, durante la transmisión del noticiero televisivo. La cuenta en sí misma, sin embargo, no tiene tanta actividad durante el noticiero como después del mismo, de 10 a 11 de la mañana. En el caso de Leo Zuckermann y Guillermo Ochoa Jr., la diferencia es aún más pronunciada.

El el caso de Laura Barrera (figura 17) es similar: su cuenta tiene la hora de mayor actividad durante el Nocitiero que presenta en Canal 22 (11.31\%), pero no tiene un pico de actividad durante la hora de su programa semanal El Soundtrack de una Vida a la 1 p.m. (4.04\%). Las interacciones dirigidas a su cuenta, por otro lado, sí tienen un pico de actividad durante dicho programa $(8.36 \%)$, equivalente al que tienen durante el noticiero $(8.81 \%)$.

El caso de Danielle Dithurbide (figura 18) es particular: hay un pico claro de interacciones de 3 a 4 de la tarde, que no coincide ni con los picos de publicación ni con el horarios de transmisión de su programa matutino en Las Estrellas, titulado Las Noticias. Una revisión de los tweets dirigidos o mencionando a la periodistas a esa hora específica nos reveló que la gran mayoría de ellos ( específicamente el 90.77\%, como se puede apreciar en la tabla 4) fueron emitidos el 21 de septiembre, en el momento del desengaño público respecto a la existencia de Frida Sofía. En la sección de discusión se retomará este asunto, ya que requiere de un contexto más amplio.

Los casos de Oscar Mario Beteta (figura 19) y Adriana Pérez Cañedo (figura 20) son los únicos en los que hay programas que no son acompañados de picos de interacción ni publicación. Como se puede apreciar en laa figura 20, la cuenta de Adriana Pérez Cañedo rigurosamente se encuentra activa sólo durante su noticiero televisivo de la tarde. En cuanto a las interacciones, hay un pequeño aumento de actividad (6.71\%) de las 9 a las 10 de la noche, pero no es comparable con el pico de la actividad de interacciones de la 1 a las 2 de la tarde (24.15\%). En el caso de Oscar Mario Beteta, sucede algo similar, aunque la cuenta de este periodista sí continúa teniendo actividad durante el día.

\section{Discusión}

Como ya se expuso en la sección de resultados, la graficación por hora de los volúmenes anuales de publicación e interacciones de las cuentas observadas nos arrojaron cuatro tipos 
de casos diferentes: en el primer tipo de caso (que comprende a 8 de los 20 observados), las publicaciones e interacciones coinciden en su comportamiento, y coinciden además con los horarios de transmisión de los noticieros de cada uno de los periodistas en cuestión. Esto pareciera intuitivo: no es de asombrar que una cuenta de Twitter, sea de quien sea, reciba más menciones y respuestas en la hora en la que más tweets publica. Tampoco es sorpresivo que los momentos en que se encuentran más activas la cuentas de los periodistas es durante la transmisión de sus respectivos programas.

Surge aquí la interrogante central del estudio: si bien podemos asumir que el aumento en publicaciones por parte de cada cuenta observada durante su noticiero se debe precisamente a la transmisión de dicho noticiero, no sabemos a esta altura si el aumento en la interacción del público con la cuenta en cuestión se debe a la actividad de la misma cuenta o a la transmisión del noticiero.

Figura 9. Actividad por hora, 2017: Mario Campos. Fuente: Elaboración propia.

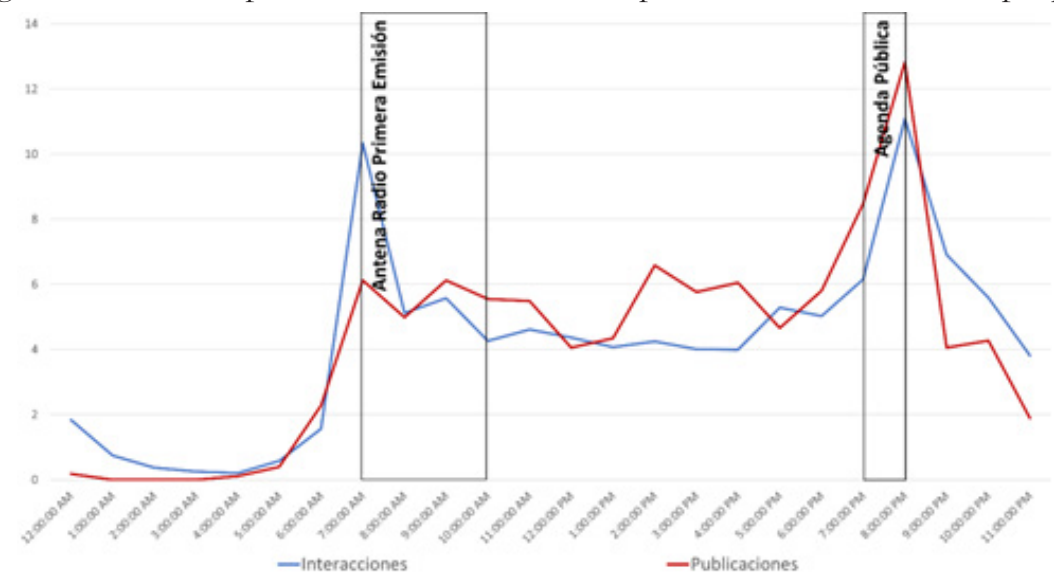

Figura 10. Actividad por hora, 2017: Ciro Gómez Leyva. Fuente: Elaboración propia.

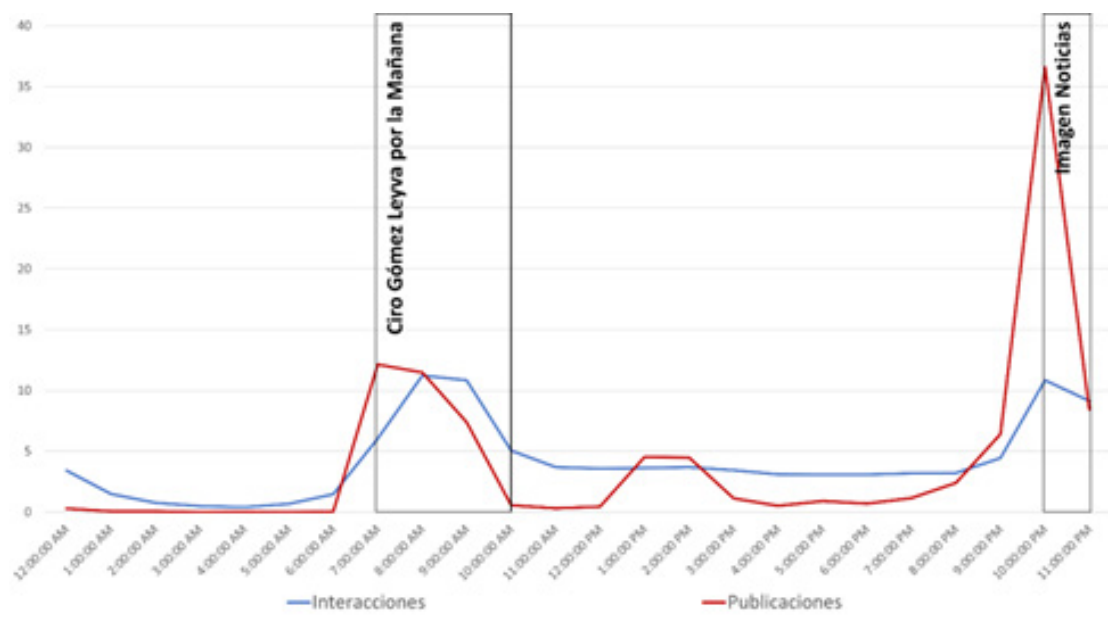


Figura 11. Actividad por hora, 2017: Carlos Loret de Mola. Fuente: Elaboración propia.

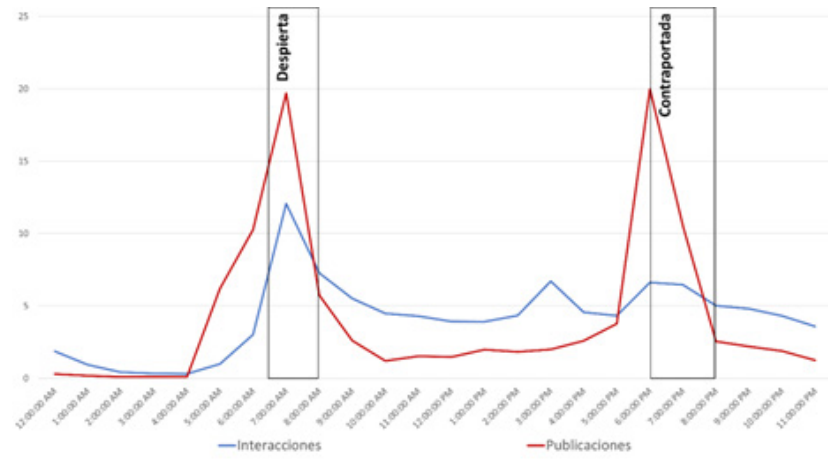

Figura 12. Actividad por hora, 2017: Denise Maerker. Fuente: Elaboración propia.

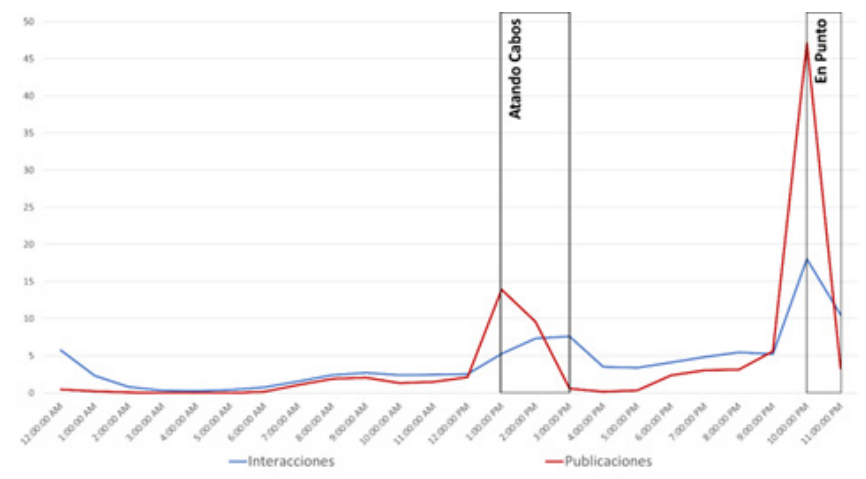

El segundo grupo de casos (5 de los 20) comienza a esclarecer esta interrogante: en este grupo se encuentran los tres periodistas que más interacciones recibieron durante el año: Carlos Loret de Mola, Denise Maerker, y Ciro Gómez Leyva. En la tabla 3 se puede observar que la diferencia en interacciones de estos tres periodistas a los que les siguen en volumen es bastante amplia (bajando de 182,602 interacciones de Ciro Gómez Leyva a 50,681 de Yuriria Sierra).

Tanto en el caso de estos periodistas como de los otros que conforman este grupo, hay diferencias de comportamiento entre las interacciones y las publicaciones; es decir, los picos en el volumen de interacciones no se encuentran en las mismas horas que los de las publicaciones, o no son tan pronunciados. Esto nos indica que la causa del aumento en interacciones no es la actividad de la cuenta, puesto que las cuentas más importantes en volumen de interacción y número de seguidores tienen horarios de alta actividad que no generan aumentos similares en la interacción.

En los casos de Denise Maerker y Carlos Loret se aprecian claramente que la actividad de sus cuentas durante sus noticieros nocturnos no está acompañada de un pico equivalente en interacciones. Podríamos suponer que la hora del día tiene algo que ver con esto; sin embargo, tenemos también casos (Mario Campos, figura 9; Leo Zuckermann, figura 15) en los que la mayor parte de la interacción sucede en el noticiero nocturno, aun cuando no haya, como en el caso de Zuckermann, actividad de la cuenta en cuestión durante esa hora.

Por otro lado, como se mencionó en los resultados, el "noticiero cool” de Javier Solórzano (figura 13) 
genera alto volumen de publicaciones durante su transmisión debido a que su formato abiertamente busca y promueve la interacción en redes sociales. Irónicamente, la cuenta de Solórzano recibe más interacción durante su noticiero tradicional, aun cuando hay menos actividad de su cuenta en ese horario. Ciro Gómez Leyva (figura 10) publica mucho más tweets durante su noticiero televisivo nocturno que durante su programa matutino de radio; sin embargo, la distribución de interacciones no refleja esta proporción, sino que se mantiene simétrica.

Los casos de este grupo nos muestran que probablemente no es la actividad en Twitter de las cuentas de los periodistas lo que provoca los aumentos en interacción, dado que podemos identificar muchos momentos en los cuales los comportamientos de los volúmenes de publicación e interacción simplemente no coinciden. El siguiente grupo nos ayuda a corroborar esta apreciación: en el caso de estos periodistas (Guillermo Ochoa Jr., Leo Zuckermann y Paola Rojas), los picos de publicación no coinciden totalmente con los noticieros, ya que dichas cuentas tienen amplia actividad de publicación fuera de los horarios de transmisión. Esta actividad no va acompañada de picos de interacción; por el contrario, éstos continúan apareciendo durante los horarios de transmisión.

En los 20 casos analizados, sólo hay dos en los cuales la transmisión de un programa no va acompañada de un pico de interacción: "Artículo Sexto" de Leo Zuckermann (figura 15) y de "Noticias de Ida y Vuelta" de Oscar Mario Beteta (figura 19). En ambos casos, no es descabellado suponer que esto se debe a la desproporcionada popularidad de los programas principales de ambos periodistas (los cuales sí generaron grandes picos de interacción) en comparación con la de los programas en cuestión. Se mantiene sin embargo la interrogante, y merece más atención en un futuro.

Figura 13. Actividad por hora, 2017: Javier Solórzano. Fuente: Elaboración propia.

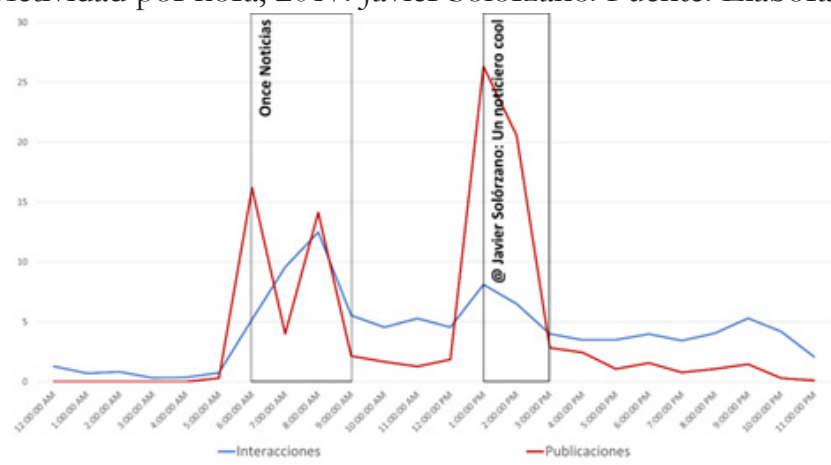

Figura 14. Actividad por hora, 2017: Guillermo Ochoa Jr.. Fuente: Elaboración propia.

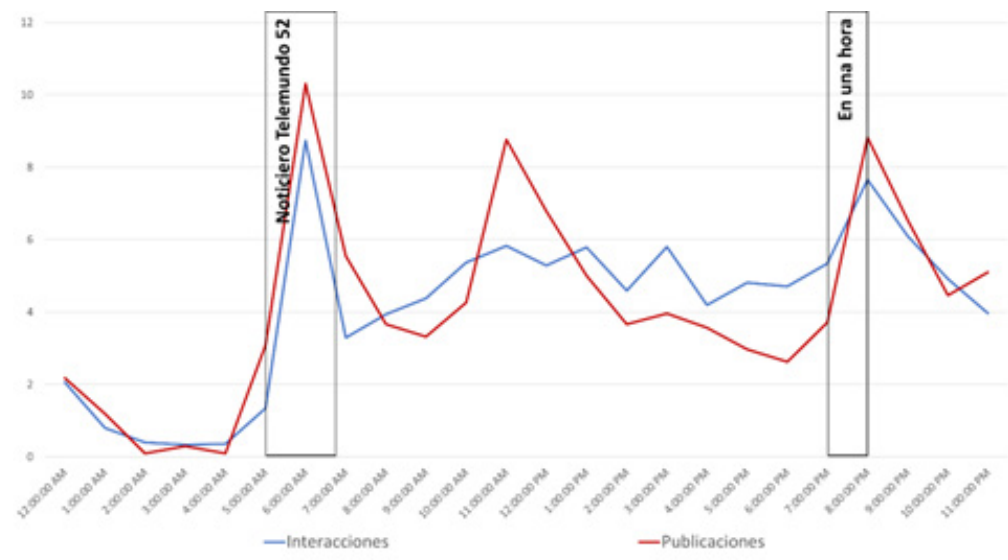


Figura 15. Actividad por hora, 2017: Leo Zuckermann. Fuente: Elaboración propia.

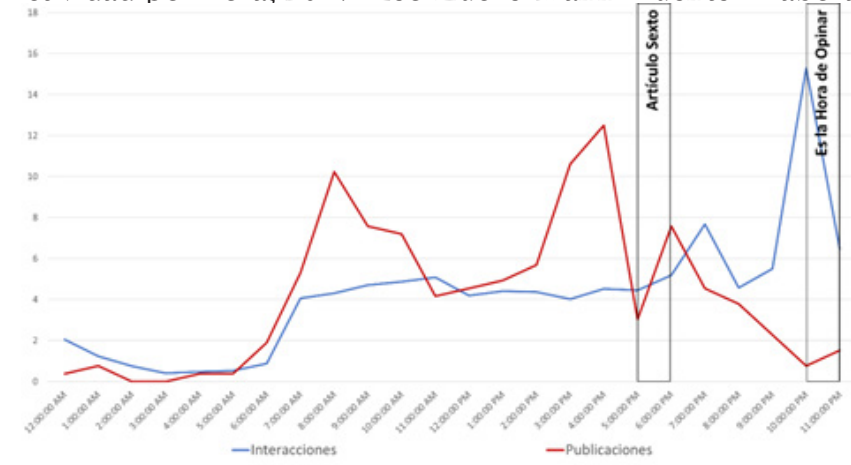

Figura 16. Actividad por hora, 2017: Paola Rojas.. Fuente: Elaboración propia.

El caso de Danielle Dithurbide resulta atípico, como va se ha mencionado, a la luz de

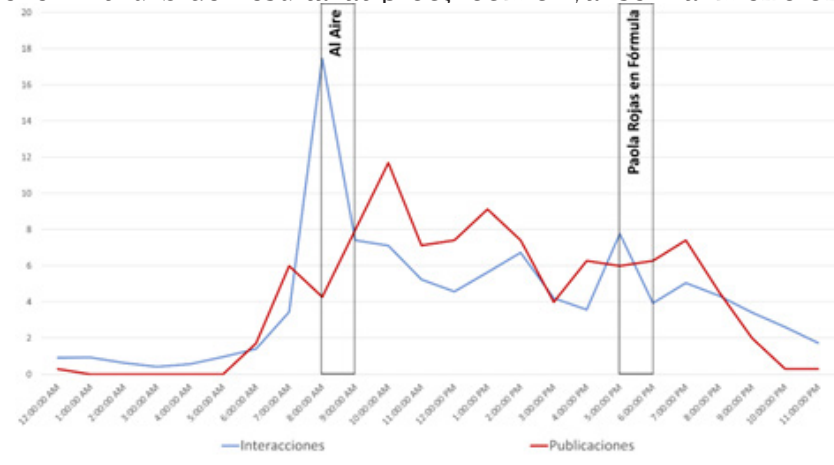

los eventos del Terremoto de Puebla (también conocido como Terremoto del 19 de septiembre). Este terremoto afectó directamente a la Ciudad de México, dejó un saldo de 369 muertos (Senado de la República, 2017) y provocó una inmediata organización y alianza de gobierno, medios y sociedad civil para el rescate y ayuda en las horas subsecuentes.

Para muchos ciudadanos, esta necesidad de organización y esfuerzo común se encarnaba en Frida Sofía, una niña enterrada bajo los escombros del derrumbado Colegio Enrique Rébsamen (Specia, 2017). Un par de días después, la población se enteró de que Frida Sofía nunca existió y su creación fue producto -en el mejor de los casos- de un error de comunicación entre la Marina, los cuerpos de rescate y los medios de comunicación (Specia, 2017). Durante los días subsecuentes, los actores involucrados se culparon unos a otros y la voz pública se enfocó en el reclamo ante la falta de profesionalismo que dio lugar a semejante confusión.

Para la opinión pública, una gran parte de la culpa recayó precisamente sobre Danielle Dithurbide, quien fungió como corresponsal de Televisa en el Colegio Rébsamen. Al revisar la tabla 4 y la figura 18, podemos comprobar que una gran mayoría de los tweets que conforman el pico de actividad de las 3 a las 4 p.m. corresponde al 21 de septiembre, fecha y hora en que se dio a conocer la inexistencia de Frida Sofía.

Al inicio de este trabajo se presentaron 3 escenarios posibles: primero, una correlación 
entre las distribuciones de publicación e interacción independientemente de los horarios de transmisión, lo cual implicaría que la interacción en Twitter alrededor de estas cuentas se debe a la actividad de la misma cuenta y no a la actividad del periodista en medios tradicionales; segundo, una correlación constante entre publicación, distribución y horarios de transmisión, lo cual impediría formular conclusiones; tercero, una correlación entre la interacción en torno a la cuenta y la transmisión de los noticieros, independientemente de la actividad de la cuenta misma, lo cual indicaría la existencia de hibridación entre el consumo mediático de noticias tradicionales y la expresión de opiniones en Twitter (es decir, un fenómeno de doble pantalla) en el ciclo de consumo de noticias de la vida cotidiana, y no solamente en momentos de alta mediatización o politización, en los cuales se ha centrado casi toda la investigación respecto al fenómeno. Se planteó un cuarto escenario, en el cual la interacción sería independiente tanto de la actividad de la cuenta del periodista como de su aparición en medios tradicionales.

Como seguramente se puede apreciar, los resultados apuntan al tercer escenario: la continua coincidencia de los noticieros radiofónicos y televisivos con las horas pico de interacción en torno a las cuentas de sus presentadores, aun cuando dichas cuentas no necesariamente están activas en esos momentos.

Esto parece indicar que, efectivamente, los fenómenos de doble pantalla o de hibridación del consumo mediático noticioso no se limitan a los momentos de gran politización como los debates electorales, sino que también forman parte de la manera en que los usuarios se relacionan con la noticia en el día a día. Claro está, no quiere esto decir que este modo de relacionarse con los medios de comunicación no se exacerbe en momentos cúspide, de crisis, de alta mediatización o movilización: si algo nos muestra el caso de Danielle Dithurbide es que los volúmenes de interacción generados en estos momentos clave superan a los cotidianos por varios órdenes de magnitud.

Figura 17. Actividad por hora, 2017: Laura Barrera.. Fuente: Elaboración propia.

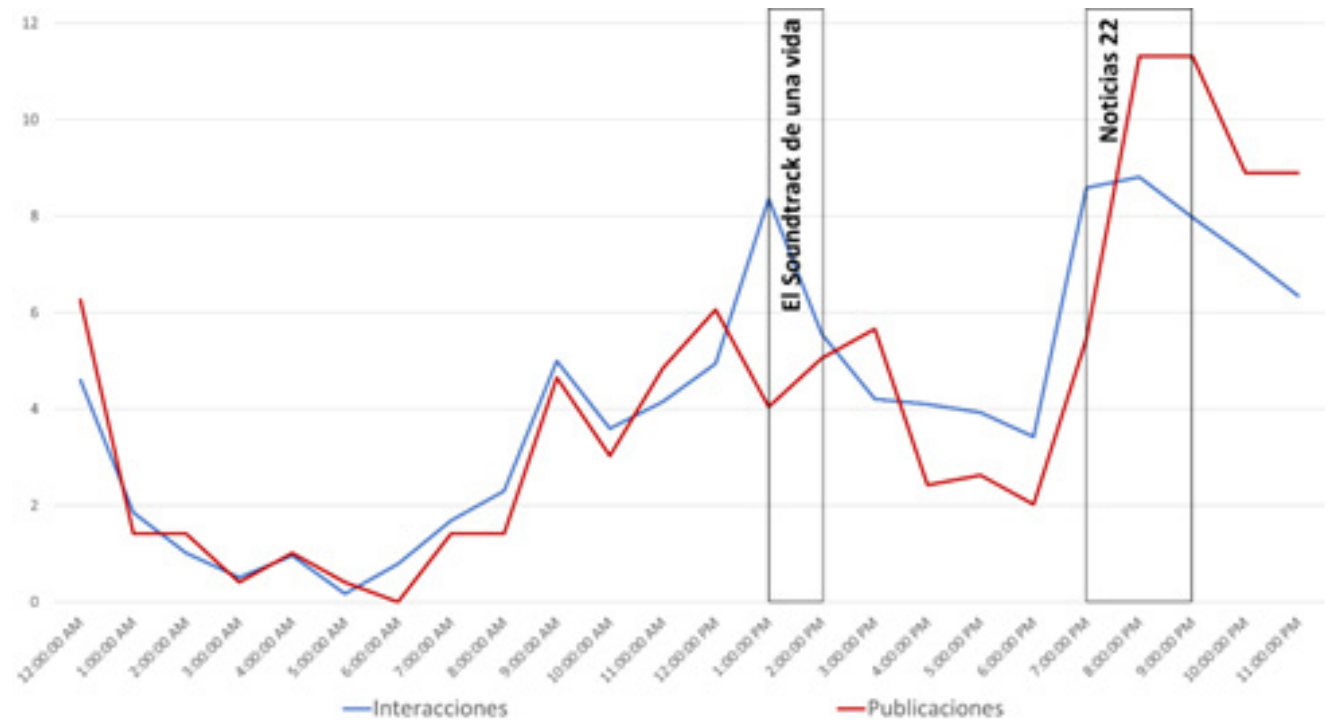


Figura 18. Actividad por hora, 2017: Danielle Dithurbide.. Fuente: Elaboración propia.

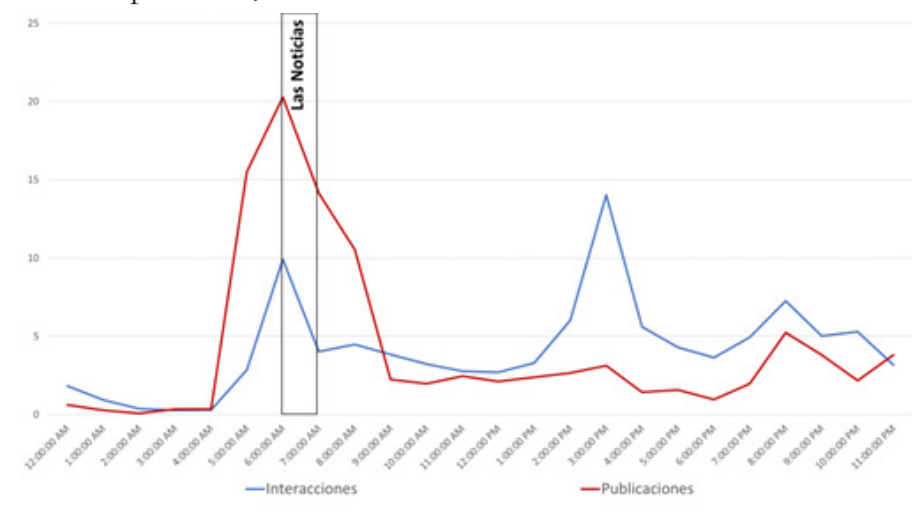

Figura 19. Actividad por hora, 2017: Oscar Mario Beteta. Fuente: Elaboración propia.

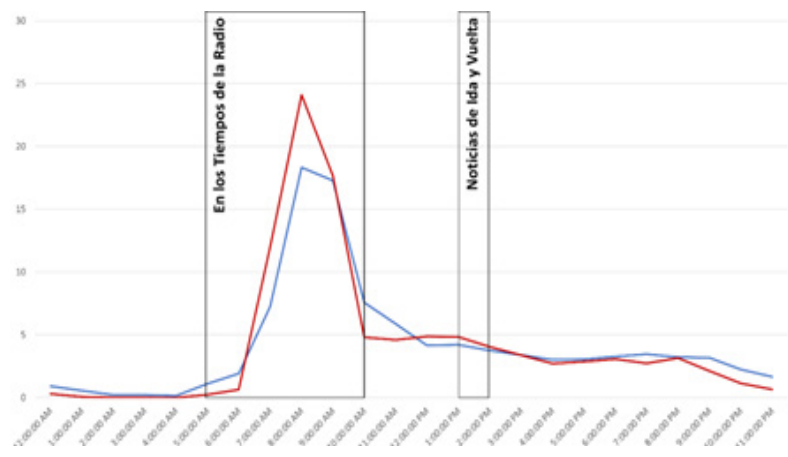

Figura 20. Actividad por hora, 2017: Adriana Pérez Cañedo. Fuente: Elaboración propia.

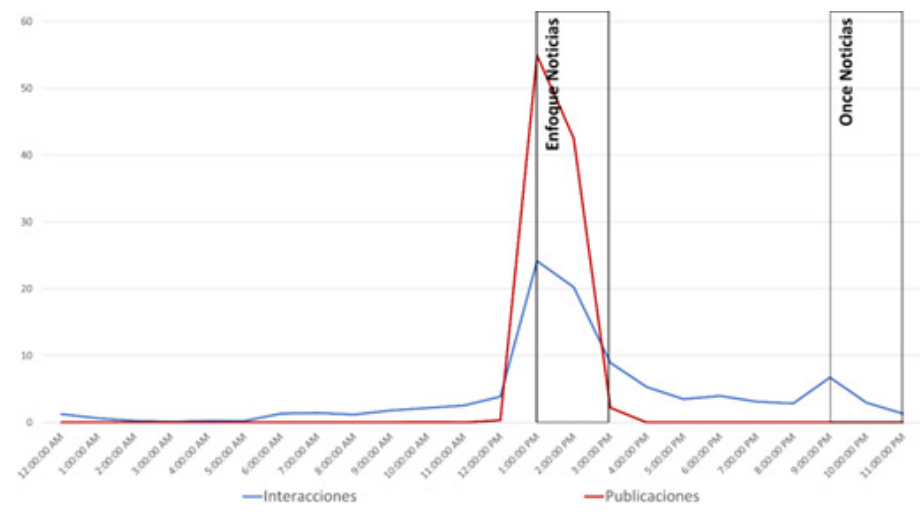


Tabla 4. Interacciones en torno a la cuenta de Danielle Dithurbide, 3:00-4:00 pm

\begin{tabular}{|l|l|l|}
\hline Día & Volumen & $\begin{array}{l}\text { Porcentaje del } \\
\text { total anual }\end{array}$ \\
\hline Septiembre 21 & 3750 & 90.77 \\
\hline Junio 29 & 56 & 1.35 \\
\hline Septiembre 20 & 39 & .94 \\
\hline Junio 21 & 22 & .53 \\
\hline Abril 23 & 14 & .34 \\
\hline Julio 20 & 13 & .31 \\
\hline Julio 02 & 11 & .27 \\
\hline Mayo 02 & 10 & .24 \\
\hline Junio 03 & 8 & .12 \\
\hline Junio 28 & 7 & .12 \\
\hline
\end{tabular}

Ahora bien: las dimensiones cambian, pero las lógicas se mantienen. Los resultados de este estudio nos ayudan a comprender que la hibridación de redes sociales y medios tradicionales no es sólo un fenómeno de los momentos clave, sino del consumo cotidiano. Esperamos que esto ayude a voltear un poco la mirada académica sobre este fenómeno de la excepcionalidad a la cotidianeidad, donde sucede la inmensa mayoría del consumo mediático y noticioso.

\section{Conclusiones}

El presente estudio recopiló durante un año natural todas las publicaciones realizadas en Twitter por -o mencionando a- 20 periodistas televisivos de México, con la finalidad de esclarecer si la interacción del público con dichas cuentas en Twitter obedecía al trabajo de los periodistas en medios tradicionales o a procesos y lógicas internos de dicha red social. El estudio encontró evidencia para asegurar, con razonable seguridad, que en el caso de estos periodistas, su actividad en medios tradicionales, y no su actividad en Twitter, es el detonante de la interacción. Esta información es de utilidad en el contexto de la discusión de los fenómenos de doble o segunda pantalla, o bien sistemas híbridos, los cuales han sido estudiados únicamente en momentos de alta politización al grado de que pudieran llegar a parecer consecuencia única de estos momentos. Los resultados de este estudio pueden servir para abrir la discusión respecto al estudio de la doble pantalla en el consumo noticioso de la vida cotidiana.

Por otro lado, el presente estudio puede servir para argumentar en contra de los términos "doble pantalla" o "segunda pantalla" en favor de un término más neutral como "sistema híbrido". Como se habrá notado en los resultados y su discusión, algunos de los noticieros que más interacción generan son radiofónicos, por lo cual pensar el fenómeno en términos de dos pantallas resulta insuficiente o, al menos, confuso.

En lo que se refiere al fenómeno en sí, se ha teorizado que la doble pantalla es predictor de 
mayor involucramiento político, pero el estudio de McGregor (2017) encontró que la relación puede ser inversa dependiendo del posicionamiento político del usuario frente a la noticia. Esto tiene todo el sentido del mundo: si decimos que no se puede pensar en la actividad en Twitter fuera del contexto de la dieta mediática del usuario, tampoco se puede pensar en dicha dieta mediática fuera del contexto de la identidad política de ciudadano. Efectivamente, observar a Twitter como un foro de discusión auto-contenido y separado del resto de la vida cotidiana no es factible: ya en investigaciones similares se ha encontrado que la discusión en Twitter no se encuentra aislada del resto de la esfera pública, y sus movimientos no puede ser explicados -como se creía en un inicio- observándola en el vacío, sin el contexto de las estructuras sociales que la informan. Tal es el caso de la relación de la actividad en Twitter con las estructuras gubernamentales (Corona y Muñoz, 2018), y con la identidad política y de clase social (Corona, 2018). Es por esto que surge la necesidad de observar a la actividad en Twitter como parte de un contexto socio-cultural, y específicamente en la cotidianeidad, donde se da la mayor parte no sólo de la interacción en Twitter, sino de la vida en general.

El siguiente paso natural pareciera ser el estudio de la recepción y sus circunstancias: hasta que no tengamos datos específicos de primera mano respecto a la utilización que el público le da a su capacidad de enunciación en Twitter, y su relación con el consumo de medios noticiosos, el conocimiento obtenido se quedará en el campo especulativo. Sería recomendable un siguiente paso cualitativo, informado por datos cuantitativos como los aquí presentados, directamente con los usuarios. Realizar investigación de esta índole nos permitiría llegar a un nivel más profundo de conocimiento sobre el fenómeno.

\section{Bibliografía}

Barberá, P., Jost, J.T., Nagler, J., Tucker, J.A. y Bonneau, R. (2015). Tweeting from left to right: Is online political communication more than an echo chamber? Psychological science, 26(10), pp. 15311542. DOI: $\underline{10.1177 / 0956797615594620}$

Bruns, A. y Highfield, T. (2012). Blogs, Twitter, and breaking news: The produsage of citizen journalism. Produsing theory in a digital world: The intersection of audiences and production in contemporary theory, 80(2012), 15-32.

Bruns, A. y Stieglitz, S. (2014). Metrics for understanding communication on Twitter. En Weller, K. et al (eds.) Twitter and Society. pp. 69-82. New York: Peter Lang.

Chadwick, A. (2017). The bybrid media system: Politics and power. Londres: Oxford University Press.

Chadwick, A., O'Loughlin, B. y Vaccari, C. (2017). Why people dual screen political debates and why it matters for democratic engagement. Journal of Broadcasting \& Electronic Media, 61(2), 220239.

Colleoni, E., Rozza, A. y Arvidsson, A. (2014). Echo chamber or public sphere? Predicting political orientation and measuring political homophily in Twitter using big data. Journal of Communication, 64(2), pp. 317-332. DOI: 10.1111/jcom.12084 
Conover, M., Ratkiewicz, J., Francisco, M., Gonçalves, B., Menczer, F. y Flammini, A. (2011). Political polarization on twitter. ICWSM, 133, pp. 89-96.

Continuum Analytics. (2012). Anaconda (Versión Custom para Python 2.7) [Software de PC]. Recuperado de https://anaconda.org/

Corona, A. (2018). Mecanismos de otrificación entre la oposición política en Twitter durante las elecciones estatales de 2017 en México. adComunica. Revista Científica de Estrategias, Tendencias e Innovación en Comunicación, 16, pp. 45-70. DOI: 10.6035/2174-0992.2018.16.4

Corona, A. y Muñoz, B. A. (2018). Twitter y organización partidista a nivel local durante la elección estatal de Coahuila, 2017. Question: Revista de Periodismo y Comunicación, 1(57). DOI: $\underline{10.24215 / 16696581 \mathrm{e} 021}$

De Zúñiga, G. y Liu, J.H. (2017). Second screening politics in the social media sphere: Advancing research on dual screen use in political communication with evidence from 20 countries. Journal of Broadcasting \& Electronic Media, 61(2), pp. 193-219. DOI: $\underline{10.1080 / 08838151.2017 .1309420}$

Effing, R., Van Hillegersberg, J. y Huibers, T. (2011). Social media and political participation: are Facebook, Twitter and YouTube democratizing our political systems?. Ponencia presentada en International conference on electronic participation (pp. 25-35). Berlin, Heidelberg.

Giglietto, F., Iannelli, L., Rossi, L. y Valeriani, A. (2016). Fakes, news and the election: A new taxonomy for the study of misleading information within the hybrid media system. Ponencia presentada en Convegno AssoComPol 2016 (Urbino, 15-17 Dicembre 2016)

Giglietto, F. y Selva, D. (2014). Second Screen and Participation: A Content Analysis on a Full Season Dataset of Tweets. Journal of Communication, 64(2), pp. 260-277. DOI: 10.1111/icom.12085

Gruzd, A. y Roy, J. (2014). Investigating political polarization on Twitter: A Canadian perspective. Policy \& Internet, 6(1), pp. 28-45. DOI: 10.1002/1944-2866.POI354

Loader, B. D. y Mercea, D. (Eds.). (2012). Social media and democracy: Innovations in participatory politics. Londres: Routledge.

Lockie, S. (2016). Post-truth politics and the social sciences. Environmental Sociology, 3(1), pp. 1-5. DOI: $10.1080 / 23251042.2016 .1273444$

McGrath, R. (2013). Twython (Versión 3.4.0 - 3.6.0) [Software de PC]. Recuperado de https:// github.com

Morales, A. J., Borondo, J., Lozada, J. C. y Benito, R. M. (2015). Measuring political polarization: Twitter shows the two sides of Venezuela. Chaos: An Interdisciplinary Journal of Nonlinear Science, 25(3). DOI: $10.1063 / 1.4913758$

Python Software Foundation. (2010). Python (Versión 2.7). [Software de PC]. Recuperado de https://www.python.org/download/releases/2.7/

Senado de la República. (2017). Recuento de los daños 7S y 19s: A un mes de la tragedia. Notas 
Estratégicas, 17. Instituto Belisario Domínguez.

Shah, D. V., Hanna, A., Bucy, E. P., Lassen, D. S., Van Thomme, J., Bialik, K., Yang, J.H. y Pevehouse, J. C. (2016). Dual screening during presidential debates: Political nonverbals and the volume and valence of online expression. American Behavioral Scientist, 60(14), 1816-1843.

Specia, Megan. (27 de septiembre de 2017). 'Frida Sofia': The Mexico Earthquake Victim Who Never Was. The New York Times. Recuperado de https://www.nytimes.com/2017/09/27/world/ americas/mexico-earthquake-trapped-girl.html

Suiter, Jane. (2016). Post-Truth Politics. Political Insight, 7(3), pp. 25-27. DOI: $\underline{10.1177 / 2041905816680417}$

Vaccari, C., Chadwick, A. y O’Loughlin, B. (2015). Dual Screening the Political: Media Events, Social Media, and Citizen Engagement. Journal of Communication, 65(6), pp. 1041-1061. DOI: 10.1111/icom.12187

Williams, S., Terras, M. y Warwick, C. (2013). What do people study when they study Twitter? Classifying Twitter related academic papers. Journal of Documentation, 69(3), pp. 384-410. DOI: 10.1108/JD-03-2012-0027

Yardi, S. y Boyd, D. (2010). Dynamic debates: An analysis of group polarization over time on twitter. Bulletin of Science, Technology \& Society, 30(5) pp. 316-327. DOI: $10.1177 / 0270467610380011$ 
MHCJ nº 10 (1) | Año 2019 - Artículo nº 10 (145) - Páginas 199 a 221 - mhjournal.org

\section{(c) (†)}

Licencia Creative Commons

Miguel Hernández Communication Journal

mhjournal.org

\section{Cómo citar este texto:}

Antonio Corona (2019): Doble pantalla todo el año: Interacción en torno a las cuentas de Twitter de periodistas televisivos en México, en Miguel Hernández Communication Journal, n¹0 (1), pp. 199 a 221. Universidad Miguel Hernández, UMH (Elche-Alicante). DOI: http://dx.doi.org/10.21134/mhcj.v10i0.291 